\title{
Adipose tissue-derived stem cells promote the reversion of non-alcoholic fatty liver disease: An in vivo study
}

\author{
NAISHUN LIAO ${ }^{1,2}$, FAN PAN $^{3}$, YINGCHAO WANG ${ }^{1,2}$, YOUSHI ZHENG $^{1,2}$, BO XU $^{1,2}$, WENWEI CHEN $^{1,2}$, \\ YUNZHEN GAO ${ }^{1,2}$, ZHIXIONG CAI ${ }^{1,2}$, XIAOLONG LIU ${ }^{1,2}$ and JINGFENG LIU ${ }^{1,2,4}$ \\ ${ }^{1}$ The United Innovation of Mengchao Hepatobiliary Technology Key Laboratory of Fujian Province, \\ Mengchao Hepatobiliary Hospital of Fujian Medical University, ${ }^{2}$ The Liver Center of Fujian Province, \\ Fujian Medical University, Fuzhou, Fujian 350025; ${ }^{3}$ Department of Hepatobiliary Surgery, \\ Fuzong Clinical College, Fujian Medical University, Fuzhou, Fujian 350001; \\ ${ }^{4}$ Liver Disease Center, The First Affiliated Hospital of Fujian Medical University, \\ Fuzhou, Fujian 350007, P.R. China
}

Received May 19, 2015; Accepted March 4, 2016

DOI: 10.3892/ijmm.2016.2528

\begin{abstract}
Non-alcoholic fatty liver disease (NAFLD) is the most common cause of liver injury and seriously affects human health. In the present study, we aimed to investigate whether adipose tissue-derived stem cell (ADSC) transplantation in combination with dietary modification was capable of reversing the progression of NAFLD. After establishing a rat model of NAFLD by feeding them a high-fat diet (HFD), ADSCs were transplanted via the portal vein into rats with HFD-induced NAFLD, and simultaneously fed a modified diet. Thereafter, gross liver morphology, the hepatosomatic (HSI) index and indicators of liver function, including alanine aminotransferase (ALT), aspartate aminotransferase (AST) and total bilirubin (TBIL) were evaluated. Subsequently, the serum levels of total cholesterol (TC), triglycerides (TGs) and fatty acids (FAs) were also assayed. Furthermore, H\&E and oil red $\mathrm{O}$ staining were used to confirm the pathological effects of NAFLD in the rat livers. Although dietary modification alone caused liver function to recover, ADSC transplantation in combination with dietary modification further decreased the HSI index, the serum levels of ALT, TBIL, TC, TGs, FAs, reduced lipid accumulation to normal levels, and reversed the hepatic pathological changes in the rat livers. Taken together, these findings suggest that ADSC transplantation assists
\end{abstract}

Correspondence to: Professor Xiaolong Liu or Professor Jingfeng Liu, The United Innovation of Mengchao Hepatobiliary Technology Key Laboratory of Fujian Province, Mengchao Hepatobiliary Hospital, Fujian Medical University, 312 Xihong Road, Fuzhou, Fujian 350025, P.R. China

E-mail: xiaoloong.liu@gmail.com

E-mail: drjingfeng@126.com

Key words: non-alcoholic fatty liver disease, adipose tissue-derived stem cells, high-fat diet, stem cell transplantation, dietary modification in the reversion of NAFLD by improving liver function and promoting lipid metabolism, thereby exerting hepatoprotective effects. Thus, we suggest that ADSC transplantation is a promising, potential therapeutic strategy for NAFLD treatment.

\section{Introduction}

It has been pointed out that non-alcoholic fatty liver disease (NAFLD) is the most common cause of chronic liver injury (1), and in many developed countries as much as onethird of the population exhibit steatosis $(2,3)$. The majority of patients with NAFLD remain asymptomatic, but $20 \%$ of NAFLD patients have experienced non-alcoholic steatohepatitis (NASH) that may progress to cirrhosis and hepatocellular carcinoma (4). The rapid pace of modern life, the increasing intake of high-energy-density foods and reduced physical activity has led to the increasing prevalence of obesity (5), which affects public health and results in numerous metabolic disorders, such as NAFLD, type 2 diabetes mellitus, hypertension and hyperlipidemia. Obesity, in particular, can result in a wide spectrum of liver abnormalities, ranging from hepatic steatosis to NASH, and even to cirrhosis (6), implying that obesity is therefore the main driver of the greater prevalence of NAFLD (1). Thus, preventing obesity will benefit patient recovery from NAFLD, and in the present study obesity was induced by feeding rats a high-fat diet (HFD), which is one approach to establishing an animal model of NAFLD (7-10).

For the management of NAFLD, medical treatment of metabolic risk factors such as dyslipidaemia and hypertension is required, and modifications to diet and physical activity must also be undertaken (11). However, antihyperlipidemic and antihypertensive drugs are associated with an increased risk of myodynia as well as liver and kidney damage. Thus, an effective strategy for the treatment of NAFLD warrants urgent investigation.

Regenerative medicine using adipose tissue-derived stem cells (ADSCs) provides a promising, novel strategy for the treatment of various intractable diseases, as ADSCs possess numerous advantages, including the ability to self-renew and 
multidifferentiate, their abundant availability, ease of obtainment and greater immunoregulatory ability $(12,13)$. Previous research has suggested that ADSCs ameliorate hypertension $(14,15)$, hyperlipemia and obesity $(16,17)$, and alleviate liver damage in an animal model of acute or chronic liver failure and liver fibrosis (18-20). Therefore, ADSC transplantation may be a suitable method of treating NAFLD. In the present study, we established a rat model of NAFLD by feeding rats a HFD in order to evaluate the therapeutic effect of ADSC transplantation in combination with dietary modification. Our results showed that ADSC transplantation promotes the reversion of NAFLD by improving liver function and by promoting lipid metabolism as well as hepatoprotective effects.

\section{Materials and methods}

Animals and ethics aproval. Fifty adult male Sprague-Dawley rats (weighing 180-200 g) were obtained from the Center for Animal Experiments of Fujian Medical University (license no. SCXKmin2012-0002). They were housed at a constant temperature $\left(22 \pm 2^{\circ} \mathrm{C}\right)$, with $60 \%$ relative humidity, and a 12:12 light-dark cycle. The rats had ad libitum access to food and autoclaved water. All animal procedures were approved by the Animal Ethics Committee of Fuzhou General Hospital (Fuzhu, China).

Isolation and culture of rat ADSCs. Rat ADSCs were harvested as previous described (21). Briefly, following anesthetization of the male Sprague-Dawley rats $(\mathrm{n}=2)$ using pentobarbital sodium (40 mg/kg; Merck \& Co., Inc., Whitehouse Station, NJ, USA), adipose tissues (approximately $3 \times 1.5 \times 0.5 \mathrm{~cm}$ ) were scraped from the subcutaneous inguinal region, cut into small pieces (approximately $0.1 \times 0.1 \times 0.1 \mathrm{~mm}$ ), and digested with $0.1 \%$ type I collagenase (Sigma-Aldrich, St. Louis, MO, USA) at $37^{\circ} \mathrm{C}$ for 60 min with gentle shaking. Subsequently, the digested tissue was filtered through a $100-\mu \mathrm{m}$ cell strainer, centrifuged at $400 \mathrm{x} \mathrm{g}$ for 5 min and washed twice with PBS (HyClone, Logan, UT, USA). The cell pellet was suspended with expanding medium consisting of $\alpha$-Modified Eagle's Medium ( $\alpha$-MEM; HyClone) with $20 \%$ fetal bovine serum (FBS; Life Technologies, Scoresby, Australia) supplemented with penicillin (100 U/ml; Life Technologies) and streptomycin $(100 \mu \mathrm{g} / \mathrm{ml}$; Life Technologies), and then transferred into 6-well plates (Corning Inc., Acton, MA, USA) at a density of $1 \times 10^{6} / \mathrm{ml}$, and incubated at $37^{\circ} \mathrm{C}$ with $5 \% \mathrm{CO}_{2}$. Following incubation for $24 \mathrm{~h}$, the non-adherent cells were discarded, whereas the adherent cells were further expanded in the complete medium, and medium was changed every 2 days. Once the cultured cells reached approximately $80 \%$ confluence, they were detached with $0.25 \%$ trypsin- $0.02 \%$ ethylenediaminetetraacetic acid (EDTA; Life Technologies) and passaged at a ratio of 1:3. Cells from the third to fifth passages were used in the present study.

Differentiation of ADSCs. To ensure that the cultured cells were ADSCs, the ability to differentiate into multi-lineage cells was investigated. Cells were seeded into 12-well plates (Corning Inc.) at a density of $3 \times 10^{4}$ cells/well in $1 \mathrm{ml}$ expansion medium. After reaching $80 \%$ confluence, the cells were cultured with specific induction medium as previously described (22). To induce osteogenic differentiation, the cells were cultured with osteogenic induction medium consisting of DMEM, 10\% FBS, $100 \mathrm{U} / \mathrm{ml}$ penicillin and $100 \mu \mathrm{g} / \mathrm{ml}$ streptomycin (all from Life Technologies), as well as $0.1 \mu \mathrm{M}$ dexamethasone, $50 \mu \mathrm{M}$ ascorbate-2-phosphate and $10 \mathrm{mM}$ $\beta$-glycerophosphate (all from Sigma-Aldrich). For adipogenesis, the cells were cultured with adipogenic induction medium consisting of DMEM, $10 \% \mathrm{FBS}, 100 \mathrm{U} / \mathrm{ml}$ penicillin and $100 \mu \mathrm{g} / \mathrm{ml}$ streptomycin (all from Life Technologies), as well as $0.5 \mathrm{mM}$ isobutyl-methylxanthine (IBMX), $1 \mu \mathrm{M}$ dexamethasone, $10 \mu \mathrm{M}$ insulin and $200 \mu \mathrm{M}$ indomethacin (all from Sigma-Aldrich). For chondrogenesis, the cells were cultured with chondrogenic induction medium consisting of DMEM, $1 \%$ FBS, $100 \mathrm{U} / \mathrm{ml}$ penicillin and $100 \mu \mathrm{g} / \mathrm{ml}$ streptomycin (all from Life Technologies), as well as $6.25 \mu \mathrm{g} / \mathrm{ml}$ insulin, $10 \mathrm{ng} / \mathrm{ml}$ TGF- $\beta 1,50 \mathrm{nM}$ ascorbate-2-phosphate (all from Sigma-Aldrich). Four weeks later, the cell population was stained using an oil red O staining kit (Nanjing Jiancheng Bioengineering Institute, Nanjing, China), an alizarin red $\mathrm{S}$ staining kit (Solarbio, Beijing, China) and also a toluidine blue staining kit (Nanjing Jiancheng Bioengineering Institute) respectively, according to the manufacturers' instructions.

Sphere formation assay. In order to further explore the activity of ADSCs, sphere cluster formation was analyzed as previously described with minor modifications (23). The cells $\left(2 \times 10^{4}\right)$ were seeded into 24-well ultralow-attachment culture plates (Thermo Fisher Scientific, Waltham, MA, USA) and cultured in StemPro ${ }^{\circledR}$ MSC SFM CTS ${ }^{\text {TM }}$ medium (Life Technologies). After culture for $24 \mathrm{~h}$, the spheres were visible under an inverted phase-contrast microscope (Zeiss, Oberkochen, Germany).

Flow cytometric analysis. The surface biomarkers of ADSCs were characterized by flow cytometric analysis to ensure cell quality. The adherent cells were firstly dissociated with $0.25 \%$ trypsin- $0.02 \%$ EDTA, then re-suspended in $\alpha$-MEM containing $10 \%$ FBS, and further incubated in PBS containing $5 \%$ bovine serum albumin (BSA; Sigma-Aldrich) for $20 \mathrm{~min}$ at room temperature. Subsequently, the cells were incubated with various primary antibodies as indicated for $60 \mathrm{~min}$ at room temperature, including phycoerythrin (PE)-conjugated anti-mouse/rat CD29 (monoclonal, 1:200) (cat. no. 12-0291-82; purchased from eBioscience, Inc., San Diego, CA, USA); mouse anti-rat/human CD31 (monoclonal, 1:100) (cat. no. SC-80913), mouse anti-rat/human CD34 (monoclonal, 1:100) (cat. no. SC-7324) (both from Santa Cruz Biotechnology, Inc., Dallas, TX, USA); PE-conjugated anti-mouse/rat CD44H (monoclonal, 1:200) (cat. no. 12-0444-82; eBioscience, Inc.), rabbit anti-rat/human/mouse CD45 (polyclonal, 1:100) (cat. no. SC-25590), goat anti-rat/human/mouse CD73 (polyclonal, 1:100) (cat. no. SC-14684), rabbit anti-rat/human/mouse CD90 (polyclonal, 1:100) (cat.no. SC-9163) (all from Santa Cruz Biotechnology, Inc.); mouse anti-rat/human CD105 (monoclonal, 1:500) (cat. no. Ab 156756) and mouse anti-rat human leukocyte antigen - antigen D related (HLA-DR) (monoclonal, 1:300) (cat. no. Ab 119795) (both from Abcam, Cambridge, UK), respectively. The cells were then washed twice with PBS, and further incubated with fluorescent-conjugated secondary antibodies donkey anti-mouse IgG-Alexa Fluor ${ }^{\circledR} 488$ (polyclonal, 1:1,000) (cat. no. A-21202) and donkey anti-rabbit IgG-Alexa Fluor ${ }^{\circledR} 647$ (polyclonal, 1:1,000) (cat. no. A-31573) (both from Invitrogen Life Technologies, Carlsbad, CA, USA) 
for $30 \mathrm{~min}$ at room temperature. Finally, the cells were washed twice with PBS and characterized using a fluorescence activated cell sorter (FACS; BD Biosciences, Franklin Lakes, NJ, USA), and the raw data were further analyzed using FlowJo 7.6 software (Tree Star, Inc., Ashland, OR, USA).

Establishing a rat model of NAFLD, and transplantation of ADSCs. Forty-eight Sprague-Dawley rats were fed either normal chow or a HFD consisting of $88 \%$ normal chow, $2 \%$ cholesterol and $10 \%$ lard, for 6 weeks. After that, the normal rats or those with NAFLD were sacrificed $(n=6)$. Following the development of hepatic steatosis, which was verified by gross examination and pathological assessment, the rats with HFD-induced NAFLD $(n=36)$ were randomly divided into two groups: the mock group $(n=18)$ which was treated with PBS ( $1 \mathrm{ml} / \mathrm{rat})$, and the ADSC therapy group $(\mathrm{n}=18)$ that received intrahepatic transplantation of ADSCs $\left(2 \times 10^{6}\right.$ cells/rat). The transplantation procedure was performed under aseptic conditions as follows: the rats were temporarily anesthetized via inhalation of ether (Sinopharm Chemical Reagent Co., Ltd., Shanghai, China), and the abdominal cavity was then opened under aseptic conditions. The portal vein was exposed with moistened swabs; subsequently, the ADSCs suspended in $1 \mathrm{ml}$ of PBS were injected into the portal vein with a 24-gauge needle. Following transplantation, all rats with HFD-induced were simultaneously treated with a modified diet (they were fed normal chow). After rats were sacrificed on weeks 2, 4 and 8 after transplantation, liver tissues (approximately $400 \mathrm{mg} / \mathrm{rat}$ ) and sera (approximately $3 \mathrm{ml} / \mathrm{rat}$ ) were collected for further investigation.

Histopathological assessment. After the rats were sacrificed using pentobarbital sodium (80 mg/kg; Merck \& Co., Inc.), the liver samples were removed and examined using a single lens reflex (SLR) camera (Nikon, Tokyo, Japan). We also measured the body and liver weight using an electronic balance (Sartorius, Goettingen, Germany) to examine the hepatosomatic index (HSI = liver weight/body weight) $\mathrm{x} 100$. Subsequently, fresh liver tissues were fixed in $4 \%$ paraformaldehyde at room temperature for $24 \mathrm{~h}$, and then gradually dehydrated with ethanol and embedded in paraffin. The paraffin blocks were subsequently sectioned $(5 \mu \mathrm{m})$ and stained with a hematoxylin and eosin (H\&E) staining kit (Nanjing Jiancheng Bioengineering Institute). Double-blind evaluation of hepatic steatosis was performed by two expert pathologists. To further clarify the degree of hepatic lipid accumulation, oil red $\mathrm{O}$ staining of the sections was performed using an oil red $\mathrm{O}$ staining kit (Nanjing Jiancheng Bioengineering Institute) following the manufacturer's instructions. The histopathological examination was performed using an inverted phase-contrast microscope (Zeiss).

Measuring serum markers of hepatic damage. To determine whether ADSC transplantation improved liver function, the sera were separated by centrifugation at $1,000 \mathrm{xg}$ for $10 \mathrm{~min}$ at $4^{\circ} \mathrm{C}$, and stored at $-80^{\circ} \mathrm{C}$. Subsequently, alanine aminotransferase (ALT), aspartate aminotransferase (AST) and total bilirubin (TBIL) were measured in the rat sera using ALT, AST and TBIL assay kits respectively, (all from Nanjing Jiancheng Bioengineering Institute) according to the manufacturer's instructions.
Measurement of serum markers of lipids metabolism. To study lipid metabolism after ADSC transplantation, the serum levels of total cholesterol (TC), triglycerides (TGs) and fatty acids (FAs) were respectively measured in rat sera using TC, TG and FA assay kits (Nanjing Jiancheng Bioengineering Institute), following the manufacturer's protocols.

Measuring hepatic markers of oxidative stress. To further explore the protective effects of ADSC transplantation on hepatic lipid peroxidation, each of the liver tissue samples were homogenized in stroke-physiological saline solution to obtain $10 \%(\mathrm{w} / \mathrm{v})$ liver homogenate, and then centrifuged at 5,000 x g for $10 \mathrm{~min}$ at $4^{\circ} \mathrm{C}$. The supernatants were subsequently collected, and hepatic superoxide dismutase (SOD) activity and malondialdehyde (MDA) content were measured using SOD and MDA assay kits (Nanjing Jiancheng Bioengineering Institute) respectively, according to the manufacturer's instructions. SOD activity and MDA content were finally normalized to total protein, which was measured using a bicinchoninic acid (BCA) assay kit (Beijing TransGen Biotech Co., Ltd., Beijing, China).

Statistical analysis. All quantitative data are expressed as the means \pm standard deviation (SD) and the statistical significance of the difference between groups was analyzed using the Student's t-test. A p-value $<0.05$ was considered to indicate a statistically significant difference.

\section{Results}

Characterization of ADSCs. Flow cytometric analysis revealed that cultured ADSCs were positive for CD29, CD44, CD73, CD90 and CD105, and negative for CD31, CD34, CD45 and HLA-DR (Fig. 1A). In relation to the adherent cells, we noted homogeneous distribution and fibroblastic shape, which is consistent with the morphological characteristics of ADSCs from other species (Fig. 1B), as has been previously described (21). These cells also had the ability to differentiate into osteogenic, adipogenic and chondrogenic lineages (Fig. 1C-E), which is a typical characteristic of mesenchymal stem cells (MSCs). Moreover, the cultured cells had the potential to form spheres (Fig. 1F), which is consistent with the results of a previous study (23). Taken together, these findings indicate that derived cells exhibited typical characteristics of ADSCs; therefore these ADSCs were subsequently used for cell transplantation.

ADSC transplantation affects gross liver morphology and the hepatosomatic index (HSI). Images of the liver samples isolated from the normal (control) group and the rats with HFD-induced NAFLD were captured to investigate the extent of liver injury, using an SLR camera (Nikon). The livers from the rats with NAFLD exhibited typical signs of hepatic steatosis, with more yellow and rough surfaces compared with the livers from the control rats. However, following dietary modification and ADSC transplantation, the gross hepatic morphology of the ADSC-treated rats showed obvious signs of recovery (Fig. 2A). Even in the mock group (rats with NAFLD treated with PBS), the signs of hepatic steatosis were alleviated, although not as markedly as the ADSC-treated group. Although dietary modification contributed to recovery from NAFLD, ADSC transplantation was an efficient strategy to accelerate liver recovery. 

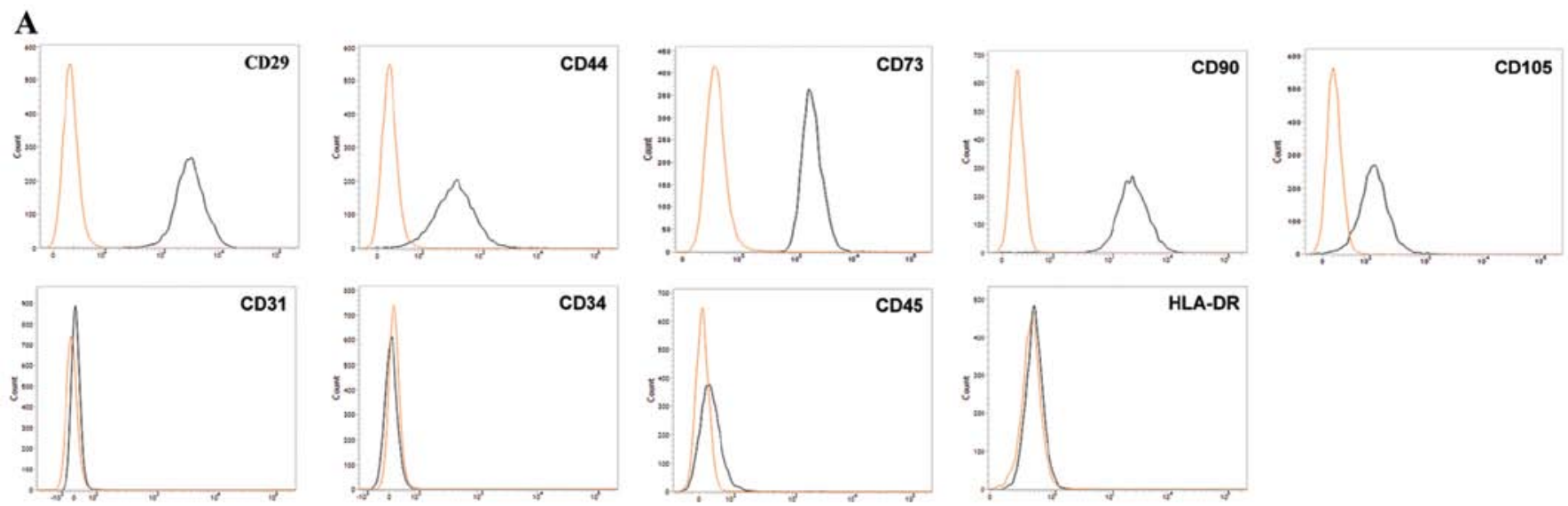

B

C

D

E

$\mathbf{F}$
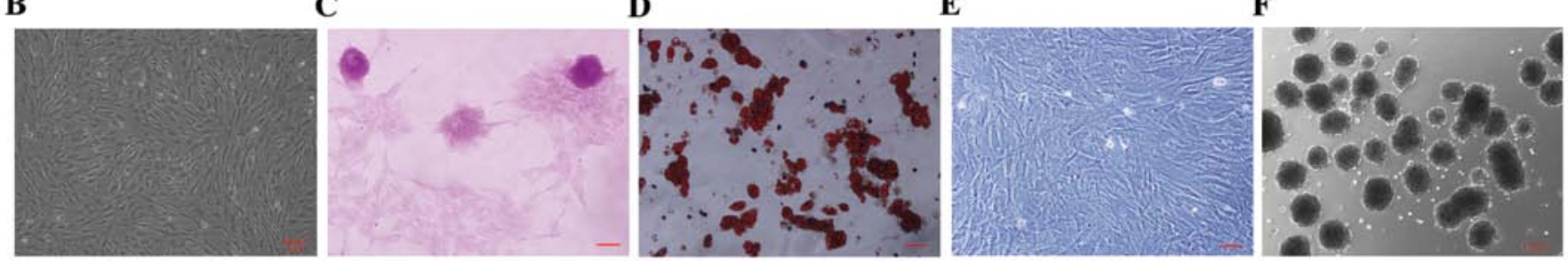

Figure 1. Characterization of rat adipose tissue-derived stem cells (ADSCs). (A) Flow cytometric analysis revealed that rat ADSCs positively expressed CD29, CD44, CD73, CD90 and CD105, and negatively expressed CD31, CD34, CD45 and human leukocyte antigen - antigen D related (HLA-DR). Orange lines indicate the negative control, while black lines indicate surface biomarker expression. (B) Fibroblastic morphology of ADSCs (magnification, x50); scale bar, $50 \mu \mathrm{m}$. (C) Osteogenic, (D) adipogenic and (E) chondrogenic differentiation of ADSCs (magnification, x200); scale bar, $20 \mu \mathrm{m}$. (F) ADSCs had the potential to form spheres (magnification, x100); scale bar, $50 \mu \mathrm{m}$.

A
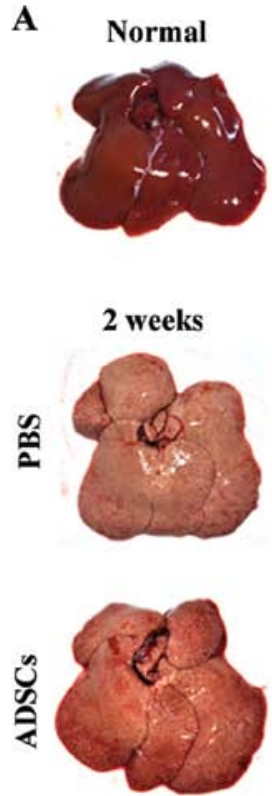

Model

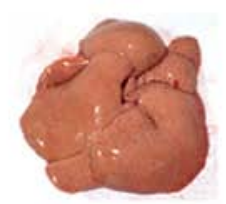

4 weeks
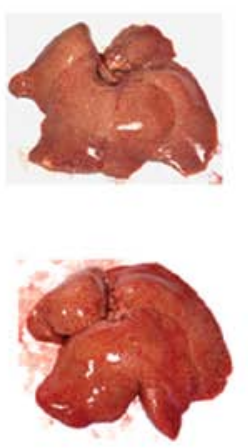
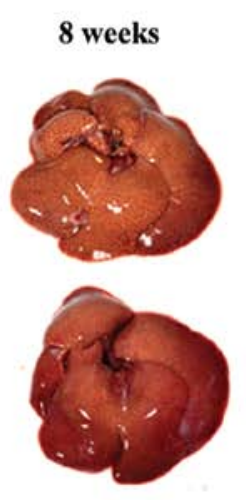

B

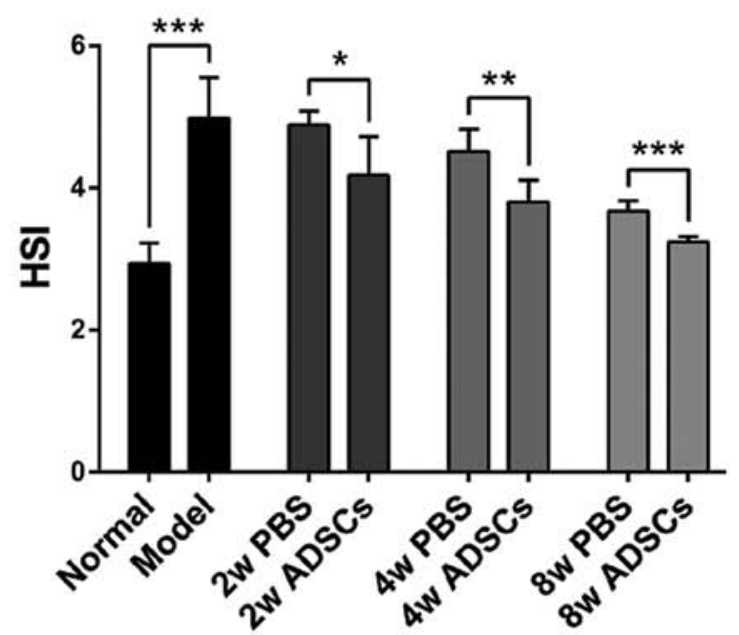

Figure 2. Adipose tissue-derived stem cell (ADSC) transplantation affects hepatic gross morphology and the hepatosomatic (HSI) index. (A) Livers harvested from the model group showed typical signs of steatosis, with more yellow and rough surfaces comparing with those of the normal rats; while ADSC transplantation induced greater reversion of the hepatic gross morphology. (B) HSI index was significantly increased in the model group compared with the normal group; while lower HSI was observed in the ADSC-treated groups compared with the mock (PBS) groups. For all groups, $\mathrm{n}=6$. ${ }^{*} \mathrm{P}<0.05,{ }^{* *} \mathrm{P}<0.01$ and ${ }^{* * *} \mathrm{P}<0.001$. Normal, untreated rats; model, rats with non-alcoholic fatty liver disease (NAFLD); $2 \mathrm{w} / 4 \mathrm{w} / 8 \mathrm{w} \mathrm{PBS}, 2 / 4 / 8 \mathrm{weeks}$ after intrahepatic transplantation of PBS into rats with NAFLD; 2w/4w/8 ADSCs, 2/4/8 weeks after intrahepatic transplantation of ADSCs into rats with NAFLD.

Furthermore, the HSI index of the rats with HFD-induced NAFLD was analyzed (the percentage of wet liver weight/body weight). The HSI of the NAFLD group was significantly higher than of the normal (control) rats, whereas the HSI decreased slightly following dietary modification, which suggested that dietary modification promoted recovery from NAFLD. Furthermore, in the ADSC-treated groups we noted a significant decrease compared to the mock groups, which 

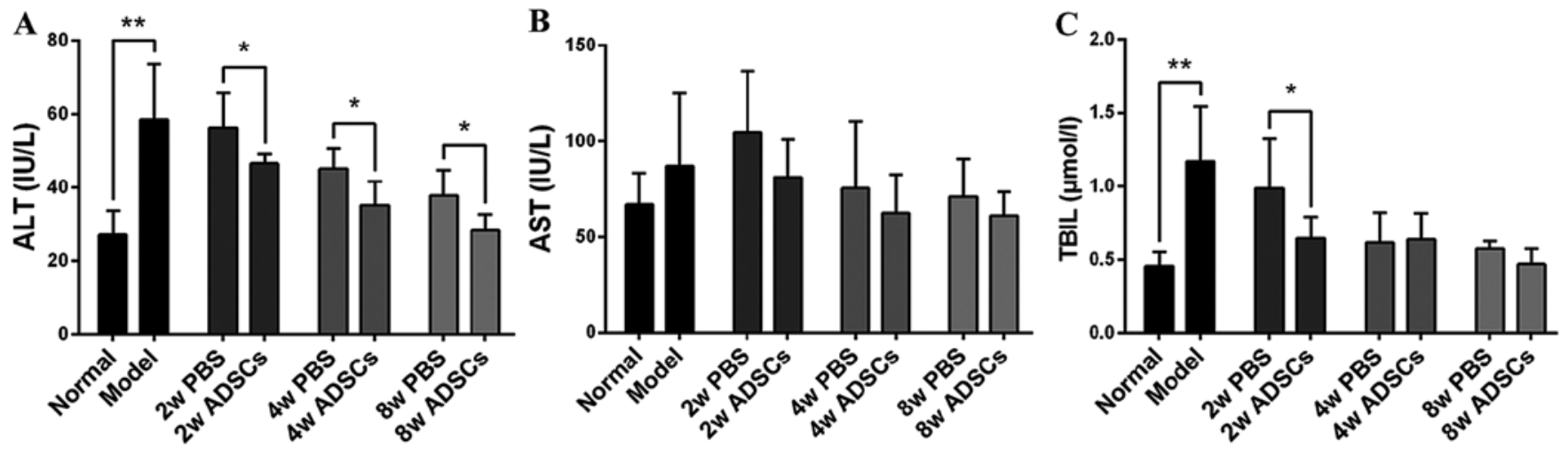

Figure 3. Adipose tissue-derived stem cell (ADSC) transplantation protects against high-fat diet (HFD)-induced liver injury in rats. (A) The alanine aminotransferase (ALT) level was significantly increased in the model group compared with that in the normal group; while a lower ALT level was observed in the ADSC-treated rats compared with the mock (PBS-treated) rats. (B) No significant difference in aspartate aminotransferase (AST) levels was observed between all groups. (C) Higher total bilirubin (TBIL) levels were observed in the model group compared with the normal group; 2 weeks after ADSC transplantation, the TBIL serum level decreased compared with that in the mock (PBS-treated) group; while there was no significant difference in the TBIL levels between the ADSC-treated groups and the mock (PBS-treated) groups 4 or 8 weeks after ADSC transplantation. For all groups, $\mathrm{n}=6$. ${ }^{*} \mathrm{P}<0.05$ and ${ }^{* *} \mathrm{P}<0.01$. Normal, untreated rats; model, rats with non-alcoholic fatty liver disease (NAFLD); $2 \mathrm{w} / 4 \mathrm{w} / 8 \mathrm{w}$ PBS, 2/4/8 weeks after intrahepatic transplantation of PBS into rats with NAFLD; 2w/4w/8w ADSCs, 2/4/8 weeks after intrahepatic transplantation of ADSCs into rats with NAFLD.
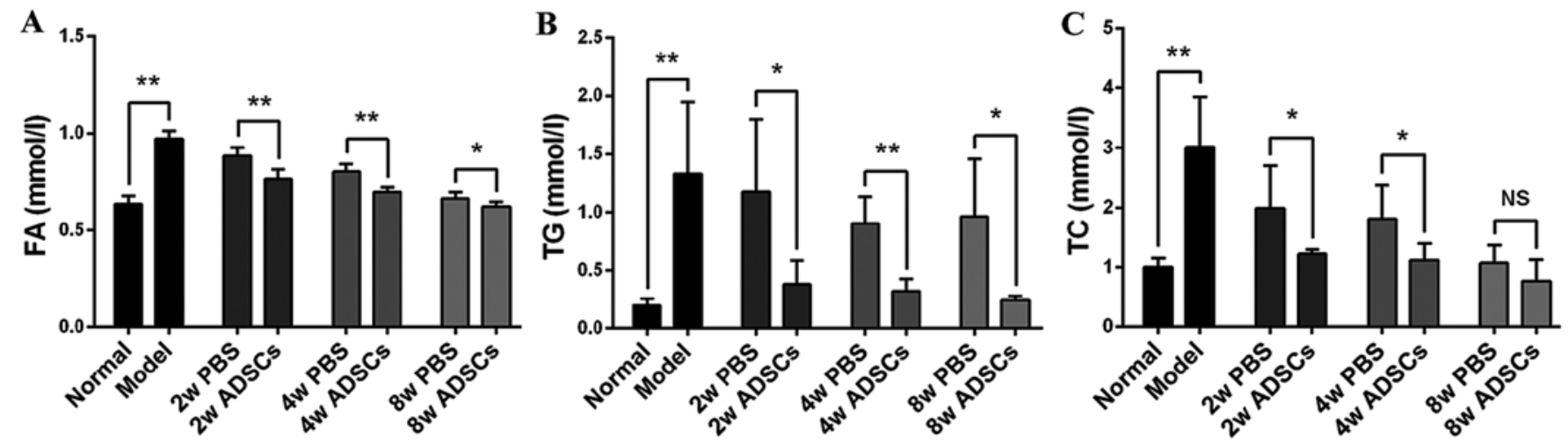

Figure 4. Adipose tissue-derived stem cell (ADSC) transplantation enhances lipid metabolism. The representative lipid metabolites of (A) fatty acids (FA), (B) triglycerides (TGs) and (C) total cholesterol (TC) were measured to evaluate the extent of lipid metabolism. Higher serum levels of FA, TG and TC were observed in the model group compared with those in the normal group; however, compared with the mock (PBS) groups, all measurements were decreased by ADSC transplantation although the TC level was slightly lower but not statistically significant (NS) 8 weeks after transplantation. For all groups, $\mathrm{n}=6$. ${ }^{*} \mathrm{P}<0.05$ and ${ }^{* *} \mathrm{P}<0.01$. Normal, untreated rats; model, rats with non-alcoholic fatty liver disease (NAFLD); $2 \mathrm{w} / 4 \mathrm{w} / 8 \mathrm{w}$ PBS, 2/4/8 weeks after intrahepatic transplantation of PBS into rats with NAFLD; $2 \mathrm{w} / 4 \mathrm{w} / 8$ ADSCs, $2 / 4 / 8$ weeks after intrahepatic transplantation of ADSCs into rats with NAFLD.

revealed that ADSC transplantation effectively reduces the liver weight of rats with NAFLD(Fig. 2B).

ADSC transplantation improves liver function. The serum levels of ALT, AST and TBIL were measured to evaluate hepatic damage in rats with HFD-induced NAFLD. Compared with the normal rats, the rats in the NAFLD group exhibited markedly increased levels of ALT and TBIL, which was an indicator of serious hepatic damage; while the AST level was slightly higher although the difference was not statistically significant. Two weeks after ADSC transplantation, the serum levels of ALT and TBIL were significantly lower than the rats in the mock group (PBS-treated rats). Furthermore, the serum levels of ALT and TBIL of the PBS-treated and ADSC-transplanted rats continuously declined when the rats were fed for another 2 (a total of 4 weeks) or 6 weeks (a total of 8 weeks), and the ADSC-transplanted rats exhibited an even lower ALT level compared with the mock rats (Fig. 3). The improvement in liver function suggested that the ADSC transplantation accelerated the recovery of the liver from NAFLD progression.

ADSC transplantation promotes lipid metabolism. In order to evaluate whether ADSCs affected lipid metabolism, serum levels of FAs, TGs and TC were measured. Compared with the normal rats, markedly higher levels of FAs, TGs and TC were observed in the NAFLD group, which means that the rats with HFD-induced NAFLD suffered from an imbalance of lipid metabolism. However, after feeding them with normal chow and administering ADSCs, the serum levels of FAs, TGs and TC in the ADSC-treated groups were significantly decreased compared to the rats treated with PBS; however, even in the mock groups improvement in the lipid metabolism was noted (Fig. 4). Thus, ADSC transplantation and dietary modification in combination affected the lipid metabolism of rats with NAFLD to the extent that levels were close to normal. Of note, 
A

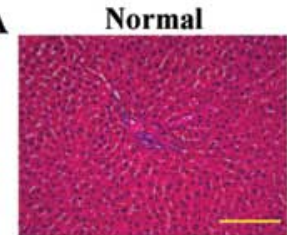

2 weeks
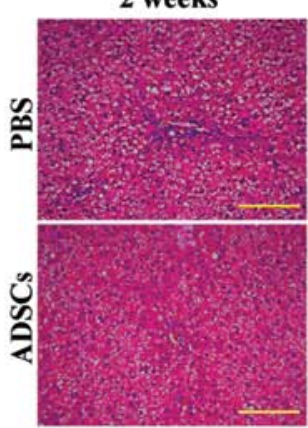

Model

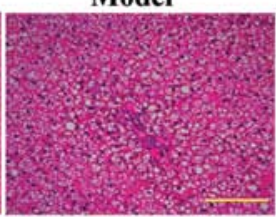

4 weeks
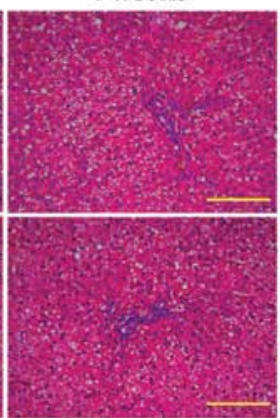

8 weeks

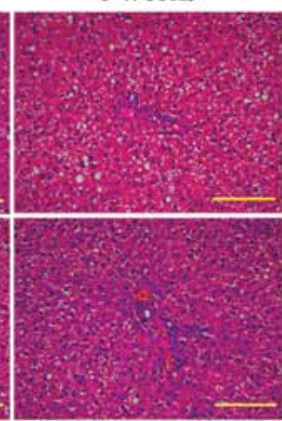

B

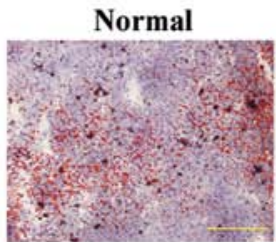

2 weeks
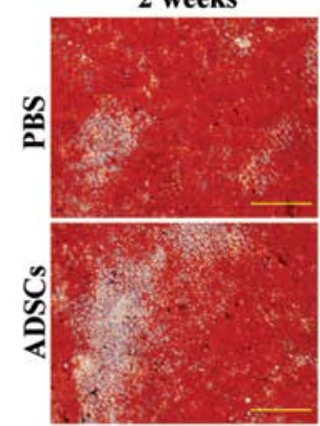

Model

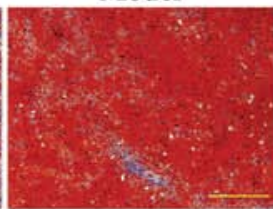

4 weeks

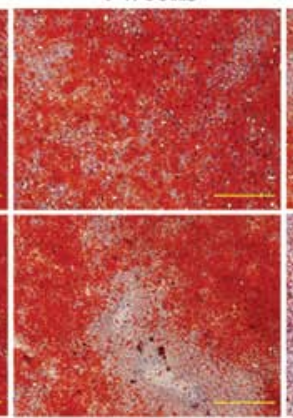

8 weeks

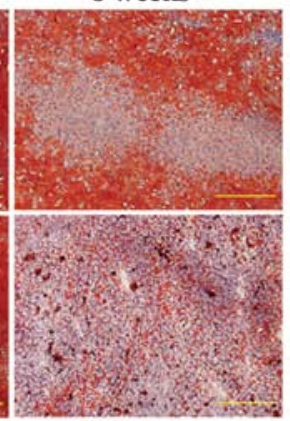

Figure 5. Adipose tissue-derived stem cell (ADSC) transplantation reverses hepatic pathological changes. The liver tissues were stained with (A) H\&E and (B) oil red O to further evaluate the protective effects of ADSCs. The liver samples collected from the normal group showed no evidence of steatosis or inflammation, while liver tissues of the rats with non-alcoholic fatty liver disease (NAFLD) (model) group exhibited signs of steatosis, with excessive lipid accumulation in the hepatocytes; however, few incidences of steatosis were observed after ADSC transplantation compared with the mock (PBS-treated) groups, and ADSC transplantation resulted in better recovery over time (magnification, $\mathrm{x} 200$; scale bar, $50 \mu \mathrm{m}$ ). Normal, untreated rats; model, rats with NAFLD.

A

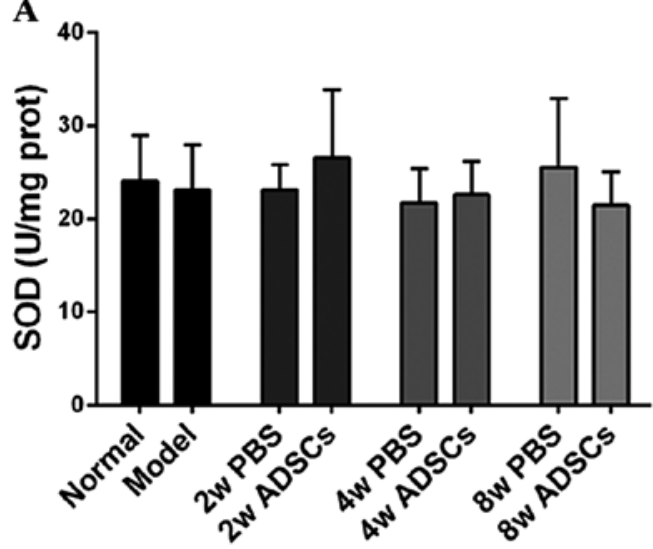

B

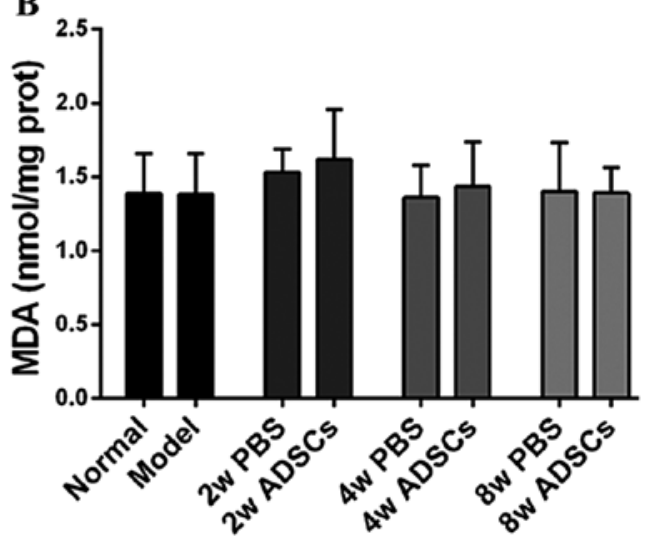

Figure 6. Absence of oxidative stress. The representative oxidative stress markers (A) superoxide dismutase (SOD) and (B) malondialdehyde (MDA) were measured to evaluate the extent of non-alcoholic fatty liver disease (NAFLD). SOD and MDA levels of the harvested liver tissues showed no significant difference between groups. Normal, untreated rats; model, rats with NAFLD; $2 \mathrm{w} / 4 \mathrm{w} / 8 \mathrm{w}$ PBS, 2/4/8 weeks after intrahepatic transplantation of PBS into rats with NAFLD; $2 \mathrm{w} / 4 \mathrm{w} / 8 \mathrm{w}$ adipose tissue-derived stem cells (ADSCs), 2/4/8 weeks after intrahepatic transplantation of ADSCs into rats with NAFLD.

at 2 and 4 weeks post-transplantation, marked improvements in lipid metablism were noted between the ADSC-treated groups and the mock groups. Furthermore, the serum levels of FAs and TGs in the ADSC-transplanted rats continued to decline when the rats were fed for another 4 weeks (a total of 8 weeks). It should be noted that there were no significant differences in the TC levels between the ADSC-transplanted group and the mock group, and the TC levels of these two groups reverted to almost normal levels (Fig. 4C). Thus, ADSC transplantation constitutes a more effective method of improving the lipid metabolism of rats with HFD-induced NAFLD.

ADSC transplantation reverses hepatic pathological changes. To further examine whether ADSC transplantation reverses the progression of NAFLD, histological examination of liver tissues was performed. The liver tissues of the normal rats exhibited no evidence of steatosis, while typical steatosis was clearly observed in the samples of rats with HFD-induced NAFLD, which means that the rat model of NAFLD was successfully established using the HFD; after dietary modification, there was less evidence of steatosis in both the PBS-treated and the ADSC-treated groups compared with the NAFLD group. Although dietary modification ameliorated steatosis in rats with HFD-induced NAFLD, fewer fat vacuoles were observed in the ADSC-treated rats compared with the mock group, which indicates that the ADSCs accelerated the reversion of NAFLD (Fig. 5A).

In order to confirm whether ADSC transplantation decreased lipid accumulation in the liver tissues of rats with NAFLD, oil red O staining was performed. Compared with the normal rats, greater lipid accumulation in the liver tissues of the NAFLD group was observed, which indicates an imbalance of lipid metabolism. Lipid accumulation was significantly 
decreased by dietary modification alone or together with ADSC transplantation, compared with the NAFLD group. Thus, we suggest that lifestyle modification alone reverses the development of NAFLD; however, we suggest that ADSC transplantation has the potential to further enhance the reversion of NAFLD since there was a marked decrease in lipid accumulation in the ADSC-treated rats compared with the mock group: lipid accumulation almost returned to normal levels in the ADSC-treated group (Fig. 5B).

Absence of oxidative stress. Excessive lipid accumulation is capable of increasing oxidative stress, which is usually characterized by low SOD activity and increased MDA content in the liver, during the progression of NAFLD. However, there was no significant difference in SOD and MDA levels in our harvested liver tissues, between all groups (Fig. 6), which indicated the absence of oxidative stress.

\section{Discussion}

NAFLD is the most common chronic liver disease in the world, and it is seriously harmful to human public health $(1,2)$. However, little is known about its pathomechanism, particularly at a cellular and a molecular level. To explain the pathological changes of NAFLD, a classical hypothesis, the 'two-hit hypothesis', was widely accepted. The 'first hit' is initiated with hepatic accumulation of high levels of free fatty acids, resulting in steatosis; this makes the liver more prone to the 'second hit', involving factors such as oxidative stress, mitochondrial dysfunction and inflammation, which lead to steatohepatitis and/or fibrosis (24,25). According to this 'two-hit hypothesis', excessive lipid accumulation may cause peroxidation, followed by increasing oxidative stress (26-28). In the present study, we therefore evaluated the SOD activity and the MDA content of the rat liver samples. However, no significant difference was observed among all the groups (Fig. 6), indicating the absence of excessive oxidative stress in our rat model; additionally, no significant difference was observed in the serum level of AST among all the groups (Fig. 3). Collectively, these data suggest that the animal model used in this study evolved in the early stages of NAFLD.

It should be noted that lifestyle modification is the key for NAFLD patients to maintain weight loss. A recent systematic review assessing the effect of diet, physical activity, and /or exercise modification in adult populations with NAFLD suggested that lifestyle modifications lead to weight reduction and consistently reduce liver fat (29). Consistent with these results, our study revealed that dietary modification moderated gross hepatic morphology and HSI index (Fig. 2), reducing hepatic damage (Fig. 3), balancing lipid metabolism and even reversing the pathological changes in the liver (Fig. 4 and 5). These results demonstrate that lifestyle modification through dietary intervention should be used to prevent the progression of NAFLD.

Although important benefits result from dietary modification, other metabolic risk factors, such as hyperlipidemia and hypertension, also require further treatment. MSC transplantation was identified as one of the choices to reduce these risk factors (14-17). Therefore, MSC transplantation presents a promising strategy for the treatment of NAFLD; previous studies have noted this in relation to bone marrow-derived stem cells (BMSCs) $(30,31)$.

As well as the common features of other MSCs, ADSCs possess the same abilities in terms of tissue repair and immune regulation (32-35), and also have many advantages, including abundant availability, ease of obtainment, better immunoregulatory ability and the fact that they are more suitable for autologous transplantation $(36,37)$. As described in this study, ADSC transplantation in combination with dietary modification was more effective at improving liver function of rats with HFD-induced NAFLD (Fig. 3), regulating lipid metabolism (Fig. 4), and ameliorating changes to the hepatic pathological morphology (Fig. 5) than treatment with dietary modification alone, which indicated that ADSC transplantation is another promising strategy for the treatment of NAFLD.

Although ADSC transplantation presents a promising therapeutic approach for the treatment of NAFLD, the mechanisms and the safety of ADSC transplantation have not yet been elucidated. Therefore, further research is necessary to ascertain this information, particularly the safety of ADSC transplantation, prior to clinical application.

In conclusion, we suggest that ADSC transplantation significantly improves liver function, promotes lipid metabolism and decreases the intrahepatic content of lipids, thereby reversing the progression of NAFLD. Therefore, ADSC transplantation presents a potential therapeutic approach for NAFLD.

\section{Acknowledgements}

This study was supported by the Key Clinical Specialty Discipline Construction Program of Fujian, China; the key project of National Science and Technology of China (grant nos. 2012ZX10002010-001-006 and 2012ZX10002016013), the National Natural Science Foundation of China (grant no. 31201008), the Key Project of Fujian Province (grant no. 2013YZ0002-3), the Science and Technology Infrastructure Construction Program of Fujian Province (grant no. 2014Y2005), the Natural Science Foundation of Fujian Province (grant nos. 2015J05175 and 2016J01592), the Project of Nanjing Military Region (grant no. 15MS136), the Scientific Foundation of Fuzhou Health Department (grant nos. 2013-S-wq15, 2013-S-wp1, 2014-S-wq-17, 2015-S-wq13 and 2014-S-wq20), and the Project of Fuzhou Science and Technology Department (grant no. 2014-S-139-3).

\section{References}

1. Sattar N, Forrest E and Preiss D: Non-alcoholic fatty liver disease. BMJ 349: g4596, 2014

2. Browning JD, Szczepaniak LS, Dobbins R, Nuremberg P, Horton JD, Cohen JC, Grundy SM and Hobbs HH: Prevalence of hepatic steatosis in an urban population in the United States: impact of ethnicity. Hepatology 40: 1387-1395, 2004.

3. Wong VW, Chu WC, Wong GL, Chan RS, Chim AM, Ong A, Yeung DK, Yiu KK, Chu SH, Woo J, et al: Prevalence of nonalcoholic fatty liver disease and advanced fibrosis in Hong Kong Chinese: a population study using proton-magnetic resonance spectroscopy and transient elastography. Gut 61: 409-415, 2012.

4. Henao-Mejia J, Elinav E, Jin C, Hao L, Mehal WZ, Strowig T, Thaiss CA, Kau AL, Eisenbarth SC, Jurczak MJ, et al: Inflammasome-mediated dysbiosis regulates progression of NAFLD and obesity. Nature 482: 179-185, 2012.

5. Lavallard VJ and Gual P: Autophagy and non-alcoholic fatty liver disease. Biomed Res Int 2014: 120179, 2014. 
6. Hassanian M, Al-Mulhim A, Al-Sabhan A, Al-Amro S, Bamehriz F, Abdo A and Al Khalidi H: The effect of bariatric surgeries on nonalcoholic fatty liver disease. Saudi J Gastroenterol 20: 270-278, 2014.

7. Lu Y, Ma Z, Zhang Z, Xiong X, Wang X, Zhang H, Shi G, Xia X, Ning $\mathrm{G}$ and $\mathrm{Li} \mathrm{X}$ : Yin Yang 1 promotes hepatic steatosis through repression of farnesoid X receptor in obese mice. Gut 63: 170-178, 2014.

8. Dhibi M, Brahmi F, Mnari A, Houas Z, Chargui I, Bchir L, Gazzah N, Alsaif MA and Hammami M: The intake of high-fat diet with different trans fatty acid levels differentially induces oxidative stress and non alcoholic fatty liver disease (NAFLD) in rats. Nutr Metab (Lond) 8: 65, 2011.

9. Wei J, Sun X, Chen Y, Li Y, Song L, Zhou Z, Xu B, Lin Y and $\mathrm{Xu}$ S: Perinatal exposure to bisphenol A exacerbates nonalcoholic steatohepatitis-like phenotype in male rat offspring fed on a high-fat diet. J Endocrinol 222: 313-325, 2014.

10. Wu J, Zhang $\mathrm{H}$, Zheng $\mathrm{H}$ and Jiang Y: Hepatic inflammation scores correlate with common carotid intima-media thickness in rats with NAFLD induced by a high-fat diet. BMC Vet Res 10 : $162,2014$.

11. Dyson JK, Anstee QM and McPherson S: Non-alcoholic fatty liver disease: a practical approach to treatment. Frontline Gastroenterol 5: 277-286, 2014.

12. Meyerrose TE, De Ugarte DA, Hofling AA, Herrbrich PE, Cordonnier TD, Shultz LD, Eagon JC, Wirthlin L, Sands MS, Hedrick MA and Nolta JA: In vivo distribution of human adipose-derived mesenchymal stem cells in novel xenotransplantation models. Stem Cells 25: 220-227, 2007.

13. Philippe B, Luc S, Valérie PB, Jérôme R, Alessandra BR and Louis C: Culture and use of mesenchymal stromal cells in phase I and II clinical trials. Stem Cells Int 2010: 503593, 2010.

14. Liu K, Liu R, Cao G, Sun H, Wangxand Wu S: Adipose-derived stromal cell autologous transplantation ameliorates pulmonary arterial hypertension induced by shunt flow in rat models. Stem Cells Dev 20: 1001-1010, 2011

15. Eirin A, Zhu XY, Krier JD, Tang H, Jordan KL, Grande JP Lerman A, Textor SC and Lerman LO: Adipose tissue-derived mesenchymal stem cells improve revascularization outcomes to restore renal function in swine atherosclerotic renal artery stenosis. Stem Cells 30: 1030-1041, 2012.

16. Okura H, Saga A, Fumimoto Y, Soeda M, Moriyama M, Moriyama H, Nagai K, Lee CM, Yamashita S, Ichinose A, et al Transplantation of human adipose tissue-derived multilineage progenitor cells reduces serum cholesterol in hyperlipidemic Watanabe rabbits. Tissue Eng Part C Methods 17: 145-154, 2011.

17. Ji AT, Chang YC, Fu YJ, Lee OK and Ho JH: Niche-dependent regulations of metabolic balance in high-fat diet-induced diabetic mice by mesenchymal stromal cells. Diabetes 64: 926-936, 2015.

18. Zhang Y, Chen XM and Sun DL: Effects of coencapsulation of hepatocytes with adipose-derived stem cells in the treatment of rats with acute-on-chronic liver failure. Int J Artif Organs 37: 133-141, 2014

19. Saito Y, Shimada M, Utsunomiya T, Ikemoto T, Yamada $S$, Morine Y, Imura S, Mori H, Sugimoto K, Iwahashi S and Asanoma M: The protective effect of adipose-derived stem cells against liver injury by trophic molecules. J Surg Res 180: 162-168, 2013.

20. Harn HJ, Lin SZ, Hung SH, Subeq YM, Li YS, Syu WS, Ding DC, Lee RP, Hsieh DK, Lin PC and Chiou TW: Adipose-derived stem cells can abrogate chemical-induced liver fibrosis and facilitate recovery of liver function. Cell Transplant 21: 2753-2764, 2012.

21. Banas A, Teratani T, Yamamoto Y, Tokuhara M, Takeshita F, Quinn G, Okochi H and Ochiya T: Adipose tissue-derived mesenchymal stem cells as a source of human hepatocytes. Hepatology 46: 219-228, 2007.

22. Zuk PA, Zhu M, Mizuno H, Huang J, Futrell JW, Katz AJ, Benhaim P, Lorenz HP and Hedrick MH: Multilineage cells from human adipose tissue: implications for cell-based therapies. Tissue Eng 7: 211-228, 2001.
23. Takahashi H, Haraguchi N, Nishikawa S, Miyazaki S, Suzuki Y, Mizushima T, Nishimura J, Takemasa I, Yamamoto $H$, Mimori K, et al: Biological and clinical availability of adiposederived stem cells for pelvic dead space repair. Stem Cells Transl Med 1: 803-810, 2012

24. Ibrahim MA, Kelleni M and Geddawy A: Nonalcoholic fatty liver disease: current and potential therapies. Life Sci 92: 114-118, 2013.

25. Shaker M, Tabbaa A, Albeldawi M and Alkhouri N: Liver transplantation for nonalcoholic fatty liver disease: new challenges and new opportunities. World J Gastroenterol 20: 5320-5330, 2014.

26. Mollica MP, Lionetti L, Moreno M, Lombardi A, De Lange P, Antonelli A, Lanni A, Cavaliere G, Barletta A and Goglia F: 3,5-diiodo-1-thyronine, by modulating mitochondrial functions, reverses hepatic fat accumulation in rats fed a high-fat diet. J Hepatol 51: 363-370, 2009.

27. Zou Y, Li J, Lu C, Wang J, Ge J, Huang Y, Zhang L and Wang Y: High-fat emulsion-induced rat model of nonalcoholic steatohepatitis. Life Sci 79: 1100-1107, 2006.

28. Takaki A, Kawai D and Yamamoto K: Molecular mechanisms and new treatment strategies for non-alcoholic steatohepatitis (NASH). Int J Mol Sci 15: 7352-7379, 2014

29. Thoma C, Day CP and Trenell MI: Lifestyle interventions for the treatment of non-alcoholic fatty liver disease in adults: a systematic review. J Hepatol 56: 255-266, 2012.

30. Ezquer M, Ezquer F, Ricca M, Allers C and Conget P: Intravenous administration of multipotent stromal cells prevents the onset of non-alcoholic steatohepatitis in obese mice with metabolic syndrome. J Hepatol 55: 1112-1120, 2011.

31. Winkler S, Borkham-Kamphorst E, Stock P, Brückner S, Dollinger M, Weiskirchen R and Christ B: Human mesenchymal stem cells towards non-alcoholic steatohepatitis in an immunodeficient mouse model. Exp Cell Res 326: 230-239, 2014.

32. Gutiérrez-Fernández M, Rodríguez-Frutos B, Ramos-Cejudo J, Teresa Vallejo-Cremades M, Fuentes B, Cerdán S and Díez-Tejedor E: Effects of intravenous administration of allogenic bone marrow- and adipose tissue-derived mesenchymal stem cells on functional recovery and brain repair markers in experimental ischemic stroke. Stem Cell Res Ther 4: 11, 2013.

33. Xie X, Wang Y, Zhao C, Guo S, Liu S, Jia W, Tuan RS and Zhang C: Comparative evaluation of MSCs from bone marrow and adipose tissue seeded in PRP-derived scaffold for cartilage regeneration. Biomaterials 33: 7008-7018, 2012.

34. Valina C, Pinkernell K, Song YH, Bai X, Sadat S, Campeau RJ, Le Jemtel TH and Alt E: Intracoronary administration of autologous adipose tissue-derived stem cells improves left ventricular function, perfusion, and remodelling after acute myocardial infarction. Eur Heart J 28: 2667-2677, 2007.

35. Niemeyer P, Kornacker M, Mehlhorn A, Seckinger A, Vohrer J, Schmal H, Kasten P, Eckstein V, Südkamp NP and Krause U: Comparison of immunological properties of bone marrow stromal cells and adipose tissue-derived stem cells before and after osteogenic differentiation in vitro. Tissue Eng 13: 111-121, 2007.

36. Sun CK, Chang CL, Lin YC, Kao YH, Chang LT, Yen CH, Shao PL, Chen CH, Leu S and Yip HK: Systemic administration of autologous adipose-derived mesenchymal stem cells alleviates hepatic ischemia-reperfusion injury in rats. Crit Care Med 40: 1279-1290, 2012.

37. Seki T, Yokoyama Y, Nagasaki H, Kokuryo T and Nagino M: Adipose tissue-derived mesenchymal stem cell transplantation promotes hepatic regeneration after hepatic ischemia-reperfusion and subsequent hepatectomy in rats. J Surg Res 178: 63-70, 2012. 